\title{
The Management of Lichen Sclerosus Urethral Strictures by 3 Different Techniques: a single-center surgical algorithm based on the evaluation of corpus spongiosum
}

\section{Dachao Zheng}

Shanghai 9th People's Hospital, Shanghai Jiaotong University School of Medicine

\section{Minkai Xie}

Shanghai 9th People's Hospital, Shanghai Jiaotong University School of Medicine

\section{Xiang Wan}

Shanghai 9th People's Hospital, Shanghai Jiaotong University School of Medicine

\section{Yuanshen Mao}

Shanghai 9th People's Hospital, Shanghai Jiaotong University School of Medicine

\section{Haijun Yao}

Shanghai 9th People's Hospital, Shanghai Jiaotong University School of Medicine

\section{Zhong Wang ( $\sim$ zhongwang2000@sina.com)}

Shanghai 9th People's Hospital, Shanghai Jiaotong University School of Medicine

\section{Research Article}

Keywords: Lichen sclerosus, spongiofibrosis, sugical algorithms, urethral stricture, urethroplasty

Posted Date: October 21st, 2021

DOl: https://doi.org/10.21203/rs.3.rs-970094/v1

License: (c) (1) This work is licensed under a Creative Commons Attribution 4.0 International License.

Read Full License 


\section{Abstract}

\section{Background}

Lichen sclerosus (LS) is a chronic, inflammatory disease of unknown cause which occurs in the male genital area and can affect the external genital and the anterior urethra. Nowdays, urethral stricture associated with LS is still a challenge to urologists and different surgical options are employed according to surgeons' personal preferences. In this study, we report our experience of surgical treatments for genital lichen sclerosus urethral stricture and try to share our surgical algorithm.

Methods

Between March 2013 and March 2018, 55 patients with LS urethral strictures underwent three different surgical techniques including of Kulkarni technique, Bracka technique and perineal urethrostomy (PU). Approaches were chosen according to the evaluation of the degree of spongiofibrosis and patients' wishes.

Results

The overall success rate was $74.55 \%$. Kulkarni technique was performed in 37 patients with a success rate of $78.38 \% .12$ patients who were planned for staged substitution urethroplasty, developed a $50 \%$ success rate, including 3 local graft contractures following the first stage, 2 fistulas and 1 dehiscence following the urethroplasty respectively. PU was chosen as the primary treatment in 6 patients and performed as salvage surgery on 4 patients, which resulted a $100 \%$ success rate. Comparing to the Kulkarni and Bracka techniques, PU offers the highest degree of success and could be considered as a final salvage for all patients and statistic difference was found between three techniques $(P=0.0082)$.

\section{Conclusion}

The staged urethroplasty by excising corpus spongiosum consumed more cost of time and money but could not provide similar therapeutic effects as the Kulkarni technique and PU. Therefore, the evaluation of corpus spongiosum is a necessary step in the surgical decision making which can make surgeons avoid unsuitable decisions and improve the success rate.

\section{Backgroud}

Lichen sclerosus (LS) is a chronic, inflammatory disease of unknown cause which occurs in the male genital area and can affect the foreskin, glans, external urethral meatus and the anterior urethra ${ }^{[1]}$. In the early process, small amounts of perimeatal skin discoloration may be only indications. With disease progression, severe phimosis and urethral stricture can result. For most urologists, LS is a difficult disease to treat due to the wide range of clinical presentations, destructive anatomic involvement, various therapeutic methods and high incidence of recurrence ${ }^{[2]}$. 
In the treatment of early LS, medical managements such as steroids and hormones have shown a certain degree of efficiency ${ }^{[3,4]}$. However, surgical managements are necessary when those drug therapies fail. Circumcision and meatoplasty play important roles in the management of early LS which limited to the glans, foreskin and external meatus ${ }^{[5,6]}$. In severe cases with long-segment urethral stricture, urethral reconstruction is necessary. Contrary to the treatments of long-segment urethral strictures caused by other diseases, genital tissue is forbidden to be used. It is a common view that nongenital tissue grafts, in especial, oral mucosa is recognized as an appropriate urethral substitute for strictures associated with LS. Three major surgical procedures including of one-stage, staged oral mucosal graft repair and perineal urethrostomy (PU) have been described with varying degrees of success ${ }^{[2,7,8]}$. However, lacking a universally agreement upon reconstructive strategy results various therapeutic opinion. Although Patel and colleagues have ever reported a large series of patients with LS who underwent surgery with these three techniques and then provided some useful options ${ }^{[9]}$, urologists still employ different surgical options according to their personal preferences.

In China, the situation is more complicated. Lacking medical popularization about LS makes patients miss their optimal therapy opportunity. To make matters worse, some urologists can even not provide specialist medical services for them. Patients with long-segment urethral strictures associated with LS underwent wrong managements like direct vision internal urethrotomy (DVIU) or genital flaps urethroplasty are always met in our center. The bad quality of urethra and corpus spongiosum after these failed surgeries makes re-operations much more difficult. In this study, we report our experience of treating the long-segment urethral strictures associated with genital LS and analyze the contributing factors which influence the success rate. We hope to provide our management algorithm for the beginners who just enter the field of urethral reconstruction.

\section{Material And Methods}

\section{Patients}

This study was approved by the ethics committee. We retrospectively reviewed the records of consecutive patients referred for urethral stricture from March 2013 to March 2018. Inclusion criteria were met by patients with urethral strictures associated with external urethral meatal stenosis and typical clinical manifestation of LS. All patients underwent surgical treatments in our department and were proven LS by biopsy. Only patients who had a minimum follow-up of 12 months were included in the study. Patients with urethral strictures caused by trauma, failed hypospadias surgeries, malignant tumors, inflammatory, iatrogenic injuries, idiopathic urethral lesions, as well as patients only requiring meatotomy were excluded. The preoperative evaluation included clinical history, physical examination, urine culture, uroflowmetry, sonourethralgraphy, retrograde and voiding cystourethrography. The objective outcome was considered a failure when any postoperative treatments were needed, including dilation and reoperation. We used the Clavien-Dindo classification to classify surgical complications.

\section{Surgical Techniques}


Patients underwent three different techniques respectively according to their different clinical characteristics, including Kulkarni technique ${ }^{[10]}$, staged substitution urethroplasty ${ }^{[11]}$ and PU ${ }^{[12]}$. Techniques were chosen according to the evaluation of the degree of spongiofibrosis, which was evaluated through the combinations of sonourethralgraphy and the theory of Chhetri ${ }^{[13]}$. When the spongiofibrosis of urethral stricture was evaluated as degree I and II, a standard Kulkarni technique was performed. If the spongiofibrosis of urethral stricture was classified into the degree III and IV, the staged substitution urethroplasty would be prioritised. The perineal urethrostomy was only performed when patient required actively.

The oral mucosal grafts were harvested from the ventral surface of the tongue (lingual mucosal graft, LMG) ${ }^{[14]}$ and/or the inner cheek (Buccal mucosal graft, BMG) ${ }^{[15]}$. LMG was the first choice in our operations and BMG was used as supplementary when the LMG was insufficient.

The size of the catheter ranged from 14-Fr to 18-Fr and they were kept for 4 weeks. All patients received intravenous antibiotics for four to seven days, followed by oral antibiotic prophylaxis until the catheter was removed. The patients' retrograde and voiding urethrography was performed 4 weeks later postoperatively. Each patient was followed-up as long as possible and uroflowmetry, urethroscopy were repeated every four months in the first year and annually thereafter. The successful reconstruction was defined as normal voiding with a peak flow $>15 \mathrm{~mL} / \mathrm{s}$, without obstructive symptoms and additional treatments.

\section{Results}

Between March 2013 and March 2018, 619 patients with urethral stricture disease were treated in our institute and 55 patients met inclusion and exclusion criteria. The patients had a median 120-month history of progressive voiding difficulty (range, 10-600 months). Patients' mean age was $50.75 \pm 12.572$ yrs (range, 21-84yrs). Of these 55 patients, 37, 12 and 6 patients planned to undergo Kulkarni technique, staged substitution urethroplasty and PU respectively. Their mean urethral defect was $9.13 \pm 4.667 \mathrm{~cm}$ (range, 3-17cm). The overall success rate with a mean follow-up of 19.13 months (range, $12-48$ months) was $74.55 \%(41 / 55)$ and postoperative complications occurred in 14 patients, which were defined as grade III (The Clavien-Dindo system). 4 patients with Kulkarni technique developed recurrent strictures: 3 were secondary to infection and one patient occurred at the proximal anastomotic site. 2 of them underwent PU as salvage surgeries, 1 patient accepted an additional Kulkarni technique and one patient refused to accept additional surgical treatments. Another 4 patients with Kulkarni technique developed meatal stenosis 3-5 months postoperatively: 3 patients voided smoothly after meatoplasty and 1 patient accepted a regular dilation and clobetasol external using. In those 12 patients who underwent staged urethroplasties, 2 of them developed fistulas and repaired after additional surgeries. Dehiscence was observed on 1 patient. Notably, local graft contracture following the first stage was observed on 3 patients then 1 patient underwent an additional operation to patch the graft and two patients required to undergo PU. No complications were observed in patients undergoing PU, including 6 patients who chose $\mathrm{PU}$ as their primary treatment and 4 patients who underwent PU as salvage surgeries. The evolutions of 
treatments are shown in Figure 1. Statistic difference of the complication rates were found between these three techniques ( $8 / 37$ VS 6/12 VS 0/6, $\mathrm{P}=0.0082$ ) and also observed between Bracka group and PU group $(6 / 11$ vs $0 / 6, P=0.0498)$. Higher complication rates were found in association with Kulkarni techinque, but there was no significant difference between Kulkarni and other two techiniques respectively (Kulkarni VS Bracka, $\mathrm{P}=0.0528$; Kulkarni VS PU, $\mathrm{P}=0.2662$ ). All donor site complications were minimal in patients. Most patients experienced minor pain and slight difficulty moving their tongue for $3-$ 7 days postoperatively. Only two patients felt slight anesthesia after 3 months of surgeries. The demographic data of patients and different techniques are shown in Table 1.

Table 1. The demographic data of patients and different techniques.

\begin{tabular}{|c|c|c|c|c|c|}
\hline & Total $(n=55)$ & Single stage $\quad(n=37)$ & Staged repair $(n=12)$ & $P U(n=6)$ & $\mathrm{p}$ \\
\hline Mean Age（y） & 50.75 & 48.73 & 53.42 & 57.83 & / \\
\hline Mean Length $(\mathrm{cm})$ & 9.13 & 7.65 & 11.25 & 14.00 & / \\
\hline Mean follow-up (m) & 19.13 & 20.05 & 20.00 & 11.67 & / \\
\hline Preoperative Mean Qmax & 6.86 & 7.39 & 5.68 & 5.98 & / \\
\hline Postoperative Mean Qmax & 21.02 & 20.41 & 20.88 & 25.12 & / \\
\hline Postoperative complications & $14(26.92 \%)$ & $8(21.62 \%)$ & $6(50.00 \%)$ & $0(0 \%)$ & $0.0082^{\circ}$ \\
\hline
\end{tabular}

\section{Discussion}

Although great progresses have been developed in urologic surgical techniques, urethral reconstruction remains a challenge for most urologists. As technology evolves, substitution urethroplasty using oral mucosal grafts has been recognized as golden standard for the treatment of anterior urethral strictures. Varying oral mucosal grafting urethroplasties including Kulkarni technique, Palminteri technique, Asopa technique, Barbagli technique and Bracka technique have been demonstrated available to the treatment of anterior stricture and resulted satisfactory outcomes in clinic practices $[10,11,16-18]$. These procedures are based on the dorsal onlay technique and have been demonstrated effective in the treatments of longsegment of LS strictures. The advantages of dorsal onlay technique are more stable and reliably wellvascularized graft bed and lower incidence of sacculation ${ }^{[19]}$. In our clinic practices, Kulkarni technique was preferred because of its high success rate (83.7\%-92\%) and wide surgical indication ${ }^{[20]}$. Comparing to other similar techniques, Kulkarni technique is a minimally invasive procedure since it can provide a maximum preserving of urethral neurovascular supply by the one-sided dissection of the entire anterior urethra ${ }^{[10]}$. Meanwhile, its surgical approach can also minimize the risk of urethrocutaneous fistula and meatus dehiscence. Moreover, Kulkarni technique has more advantages like superior muscular support of the urethra, less graft and time consuming, decreased post-micturition dribbling and preservation of ejaculatory function in the treatment of panurethral stricture ${ }^{[20]}$. 
Besides of these advantages mentioned above, our surgical decision making was also related to the degree of spongiofibrosis. We referenced from Chhetri's modified classification of urethral stricture ${ }^{[12]}$. This classification is suitable for anterior urethral stricture evaluation since it has a detailed description about the urethral mucosa and corpus spongiosum. When the fibrosis only involved the urethral mucosa (Grade I) or partial corpus spongiosum (Grade II), it meant the corpus spongiosum can maintain a certain degree of elasticity for an adequate lumen width (14-Fr catheter at least) and then Kulkarni procedure was performed. Once the stricture fibrosis involved the full thickness of corpus spongiosum (Grade III), or even with extension of fibrosis beyond the corpus spongiosum and other complications (Grade IV), the one-staged urethroplasty using dorsal onlay technique should be given up. Since the severe spongiofibrosis may cause high-tension closure of corpus spongiosum and then lead to a high incidence of surgical failures. At this situation, ventral onlay augmentation without corpus spongiosum closure should not be recommended. If we take a risk to suture the mucosal grafts laterally to the ventral corpus spongiosum directly for pursuing an adequate lumen width, lacking excellent neurovascular support of corpus spongiosum may result a high breakdown rate. Palmer reported a modified ventral onlay urethroplasty with an $80 \%$ success rate ${ }^{[21]}$. In this procedure, gracilis muscle flap was used as a graft bed. But it seems not amenable to long-segment urethral stricture associated with penile urethra.

Once patients had no opportunity to be treated by single staged urethroplasty, other surgical procedures should be provided. In patients seeking for complete preservation of urethra and standing micturition, the staged urethroplasty removing the strictured urethra entirely and replacing it with oral mucosal grafts would result more durable outcomes ${ }^{[22]}$. Bracka procedure is a classic staged urethroplasty using oral mucosal grafts and is popular in the repairs of some complex anterior defects ${ }^{[11,23]}$. The major advantage of this procedure is providing a healthy neourethral plate for urethroplasty. Disadvantages are also obvious: patients always lose the normal structures of urethra and need large consuming of graft when the defects were extremely long. As we know that the longer urethral defect is, the higher incidence of complications occurs. Actually, removing the strictured urethra entirely is a two-edged sword-patients get opportunities to recover normal micturition but should afford risks of complications like hypospadias patients who accept this procedure. Besides of those common complications like fistula, recurrence of stricture, meatus dehiscence and diverticula, patients also need to face with the focal graft contracture and/or LS recurrence following the first stage operation. Although the staged urethroplasty was demonstrated an effective solution, its success rates were still lower than those single staged procedures $[7,24]$. Even more, more time consuming and additional risks of complications prevented us from applying it as a conventional technique.

Inappropriate treatment may be the major factor causing the terrible condition of the urethral plates and corpus spongiosum. In our study, previous histories of interventions like urethral dilatation, DVIU and urethroplasty were recorded in 14 patients, whose corpus spongiosum were classified into Grade III and Grade IV. There is no doubt that interventions like DVIU and urethroplasty can damage the corpus spongiosum and lead to worse degrees of spongiofibrosis. Sometimes, dilatation can also cause the urethral mucosa rupture and urethral submucosa pseudocanal. All of these interventions are 
contradictory to the therapeutic principle of long-segment anterior urethral strictures. Lacking well and standardized training of urethral diseases may be the main reason. Proper advices or treatments may reduce the difficulty of patients' following treatments and relieve their sufferings.

According to the theory we mentioned above, the evaluation of urethral plate and corpus spongiosum is an important factor in the decision-making before surgery. Physical examination is a simple and effective method that urologists can obtain the urethral characteristics as many as possible. Grade IV strictures can be easily diagnosed because of the stiff corpus spongiosum. However, Grade II and III strictures sometimes can't not be identified easily by palpation. Thus, image examinations are utilized. Retrograde and voiding urethrography, the most conventional method of image examinations, is useless in the evaluation of spongiofibrosis and only provides a two-dimensional image to show the position and rough length of the strictures. Conversely, magnetic resonance imaging (MRI) and ultrasonograhy can provide not only the accurate morphological features like the lengths of strictures but also more rich details that are not seen on radiographic contrast-enhanced urethrography, including the conditions of urethral mucosa, corpus spongiosum ${ }^{[25,26]}$. Thus, preoperative MRI or ultrasonograhy are recommended as far as possible in the treatments of anterior urethral strictures.

Our overall success rate was not as high as similar studies but still acceptable ${ }^{[7,8,27]}$. In the patients with single staged procedures, external meatus stenosis was the major complication. Lacking experience in the reconstruction of external urethral meatus may be the main factor. Xu had advised to remove the scar tissue beneath the mucosa of meatus as thoroughly as possible and construct the new meatus in an adequately wide 3-leaf clover shape ${ }^{[8]}$. We did not take attention to these advices in our early practices and then scar contracture occurred on the newly constructed external urethral meatus. In our later operations, thorough and extended resections of the mucosa and scar tissue were carried out strictly and stenosis were not observed any more. However, the success rate of patients who underwent staged urethroplasties was unsatisfactory, no matter in the graft patching stage or urethroplasty stage. In our study, local graft contracture following the first stage was observed on 3 patients. Then, only 1 patient underwent an additional operation to patch the graft in the second operation and the other 2 patients selected to maintain the status post- 1 st stage because of their smooth voiding. Therefore, 10 patients completed the whole staged urethroplasty and finally developed 3 complications. The additional revision rate $(3 / 12,25 \%)$ and high complication rate $(6 / 12,50 \%)$ driven us to perform the staged urethroplasty just on the patients with severe spongiofibrosis in our subsequent treatments and strong requirement for standing urination, which was in accordance with Kozinn's opinion ${ }^{[28]}$. However, it's far too early to draw a conclusion that the staged urethroplasty is an unsuitable approach according to such a small sample, Kulkarni had reported a $73 \%$ success rate with a 2-stage oral mucosal graft urethroplasty. For patients with severe spongiofibrosis, 2-stage oral mucosal graft urethroplasty may be the only choice if they required for preserving their complete urethra. If patients did not mind the postoperative micturition posture, PU with high success rates may be a better choice, especially for older patients with long strictures and adverse etiology, or patients who have lost their confidence in the surgeries ${ }^{[9,29]}$. In our series, 10 patients underwent PU finally: 6 patients with long segment of strictures chose PU as their 
primary treatment, 2 patients who were planned for staged urethroplasties changed their plans, and 2 patients with failed Kulkarni techniques underwent PU as salvage surgeries. All of them got smooth postoperative voiding and were satisfactory to the results. PU could provide a solution of minimizing the risk and maximizing the benefit, which was accordance with the trends of the reconstruction of complex anterior urethral strictures ${ }^{[30]}$.

Although management algorithms for long-segment anterior urethral stricture had been proposed in many papers ${ }^{[9,20]}$, the condition of corpus spongiosum was rarely mentioned. We consider that evaluation of corpus spongiosum plays an important role in optimizing operation scheme. Therefore, we proposed a modified management algorithm (Figure 2) based on the above data. However, limitations of this study are still obvious. The major limitation was its retrospective nature, which led to a less restrictive design and selection bias. The small sample would also affect the accuracy of statistical analysis. Besides, the follow-up was not long enough for detecting the progress of LS. However, taking into consideration of the various approaches to treating long-segment of urethral stricture associated with LS, this study provides information to help the surgeon regarding treatment decisions.

\section{Conclusion}

In this study, the $74.545 \%$ overall success rate indicated LS urethral stricture remained a challenge to the reconstructive surgeon, but we still got some meaningful results from it. For most patients, Kulkarni technique is an effective treatment with satisfactory degree of success. Contrary, multi-staged urethral reconstruction should not be chosen except the severe spongiofibrosis causes a high-tension closure of corpus spongiosum. PU can provide a high success rate and is suitable to all cohorts of patients. Before making a treatment decision, the evaluation of corpus spongiosum should be considered emphatically and surgeons may avoid some unsuitable surgical decisions.

\section{Abbreviations}

\section{Lichen sclerosus}

LS

Perineal urethrostomy

PU

Magnetic resonance imaging

MRI

Direct vision internal urethrotomy

DVIU

Lingual mucosal graft

LMG

Buccal mucosal graft

BMG 


\section{Declarations}

\section{Ethics approval and consent to participate}

A statement regarding ethical approval was obtained from the ethics committee of Shanghai Ninth

People's Hospital affiliated with Shanghai JiaoTong University School of Medicine (HKDL.[2016]19). The authors state that they have obtained appropriate institutional review board approval or have followed the principles outlined in the Declaration of Helsinki for all human or animal experimental investigations. In addition, for investigations involving human subjects, informed consent has been obtained from the participants involved.

\section{Consent for publication}

Not applicable.

\section{Availability of data and materials}

The datasets used and/or analysed during the current study are available from the corresponding author on reasonable request.

\section{Competing interests}

The authors declare that they have no competing interests

\section{Funding}

This study was supported by grants from the National Natural Science Foundation of China (Grant No. 81700582) and Cross-disciplinary Research Fund of Shanghai Ninth People's Hospital, Shanghai Jiao Tong University School of Medicine (JYJC201912).

\section{Authors' contributions}

DC Zheng: Project development, Data Collection, Manuscript writing

HJ Yao: Project development, Data collection

MK Xie: Project development

$X$ Wan and YS Mao: Outpatient service, Data collection

Z Wang: Critical revisions to the manuscript regarding important intellectual content.

\section{Acknowledgements}

Thanks to the staff of the department of urology, Shanghai 9th People's Hospital for their help in data collecting for publication. 


\section{References}

[1] Pugliese J M, Morey A F, Peterson A C. Lichen Sclerosus: Review of the Literature and Current Recommendations for Management. J Urol 2007;178(6):2268-2276.

[2] Barbagli G, De Angelis M, Romano G et al. Clinical outcome and quality of life assessment in patients treated with perineal urethrostomy for anterior urethral stricture disease. J Urol 2009; 182(2): 548-557.

[3] Kiss A, Csontai A, Pirot $L$ et al. The response of balanitis xerotica obliterans to local steroid application compared with placebo in children. J Urol 2001; 165(1): 219-220.

[4] Bracco GL, Carli P, Sonni L et al: Clinical and histologic effects of topical treatments of vulval lichen sclerosus. A critical evaluation. J Reprod Med 1993; 38(1): 37-40.

[5] Depasquale I, Park AJ, Bracka A. The treatment of balanitis xerotica obliterans. BJU Int 2000; 86(4): 459-65.

[6] Morey AF, Lin HC, DeRosa CA et al. Fossa navicu-laris reconstruction: impact of stricture length on outcomes and assessment of extended meatoplasty (first stage Johanson) maneuver. J Urol 2007;177(1): 184-7.

[7] Kulkarni S, Barbagli G, Kirpekar D et al. Lichen sclerosus of the male genitalia and urethra: surgical options and results in a multicenter international experience with 215 patients. Eur Urol 2009(4); 55: 94554.

[8] Xu YM, Feng C, Sa YL et al. Outcome of 1-Stage Urethroplasty Using Oral Mucosal Grafts for the Treatment of Urethral Strictures Associated With Genital Lichen Sclerosus. Urology 2014;83(1):232-236.

[9] Patel C K, Buckley J C, Zinman L N et al. Outcomes for Management of Lichen Sclerosus Urethral Strictures by 3 Different Techniques. Urology 2016;91:215-221.

[10] Kulkarni S, Barbagli G, Sansalone S et al. One-sided anterior urethroplasty: a new dorsal onlay graft technique. BJU Int 2009; 104(8): 1150-5.

[11] Bracka A. Hypospadias repair: the two-stage alternative. Br J Urol 1995;76 suppl 3:31-41.

[12] Peterson AC, Palminteri E, Lazzeri M et al. Heroic measures may not always be justified in extensive urethral stricture due to lichen sclerosus (balanitis xerotica obliterans). Urology 2004; 64(3): 565-8.

[13] Chhetri RK, Shrestha GK, Joshi HN et al. Management of urethral strictures and their outcome. Nepal Med Coll J 2009;11(1):5-8.

[14] Barbagli G, De Angelis $M$, Romano $G$ et al. The use of lingual mucosal graft in adult anterior urethroplasty: surgical steps and short-term outcome. Eur Urol 2008;54(3):671-6. 
[15] Eppley BL, Keating M, Rink R. A buccal mucosal harvesting technique for urethral reconstruction. J Urol 1997;157(4):1268-70.

[16] Palminteri E, Manzoni G, Berdondini E et al. Combined dorsal plus ventral double buccal mucosa graft in bulbar urethral reconstruction. Eur Urol 2008; 53(1): 81-9.

[17] Asopa HS, Garg M, Singhal GG et al. Dorsal free graft urethroplasty for urethral stricture by ventral sagittal urethrotomy approach. Urology 2001;58(5):657-9.

[18] Barbagli G, Selli C, Tosto A et al. Dorsal free graft urethroplasty. J Urol 1996; 155(1): 123-6.

[19] Figler BD, Malaeb BS, Dy GW et al. Impact of graft position on failure of single-stage bulbar urethroplasties with buccal mucosa graft. Urology 2013; 82(5): 1166-70.

[20] Kulkarni SB, Joshi PM, Venkatesan K. Management of panurethral stricture disease in India. J Urol 2012; 188(3): 824-30.

[21] Palmer DA, Buckley JC, Zinman LN et al. Urethroplasty for high risk, long segment urethral strictures with ventral buccal mucosa graft and gracilis muscle flap. J Urol 2015; 193(3): 902-5.

[22] Depasquale I, Park AJ, Bracka A. The treatment of balanitis xerotica obliterans. BJU Int 2000; 86(4): 459-465.

[23] Nitkunan T, Johal N, O'Malley K et al. Secondary hypospadias repair in two stages. J Pediatr Urol 2006;2(6):559-63.

[24] Selim M, Salem S, Elsherif E et al. Outcome of staged buccal mucosal graft for repair of long segment anterior urethral stricture. BMC Urol 2019;19(1):38.

[25] Chen L, Feng C, Lv XG et al. Three-Dimensional Computerized Model Based on the Sonourethrogram: A Novel Technique to Evaluate Anterior Urethral Stricture. J Urol 2018;199(2):568-575.

[26] Ryu J, Kim B. MR imaging of the male and female urethra. Radiographics 2001;21(5):1169-85.

[27] Levine LA, Strom KH, Lux MM. Buccal mucosa graft urethroplasty for anterior urethral stricture repair: evaluation of the impact of stricture location and lichen sclerosus on surgical outcome. J Urol 2007;178(5): 2011-2015.

[28] Kozinn SI, Harty NJ, Zinman L et al. Management of complex anterior urethral strictures with multistage buccal mucosa graft reconstruction. Urology 2013;82(3): 718-22.

[29] Fuchs JS, Shakir N, McKibben MJ et al. Changing Trends in Reconstruction of Complex Anterior Urethral Strictures: From Skin Flap to Perineal Urethrostomy. Urology 2018;122:169-173. 


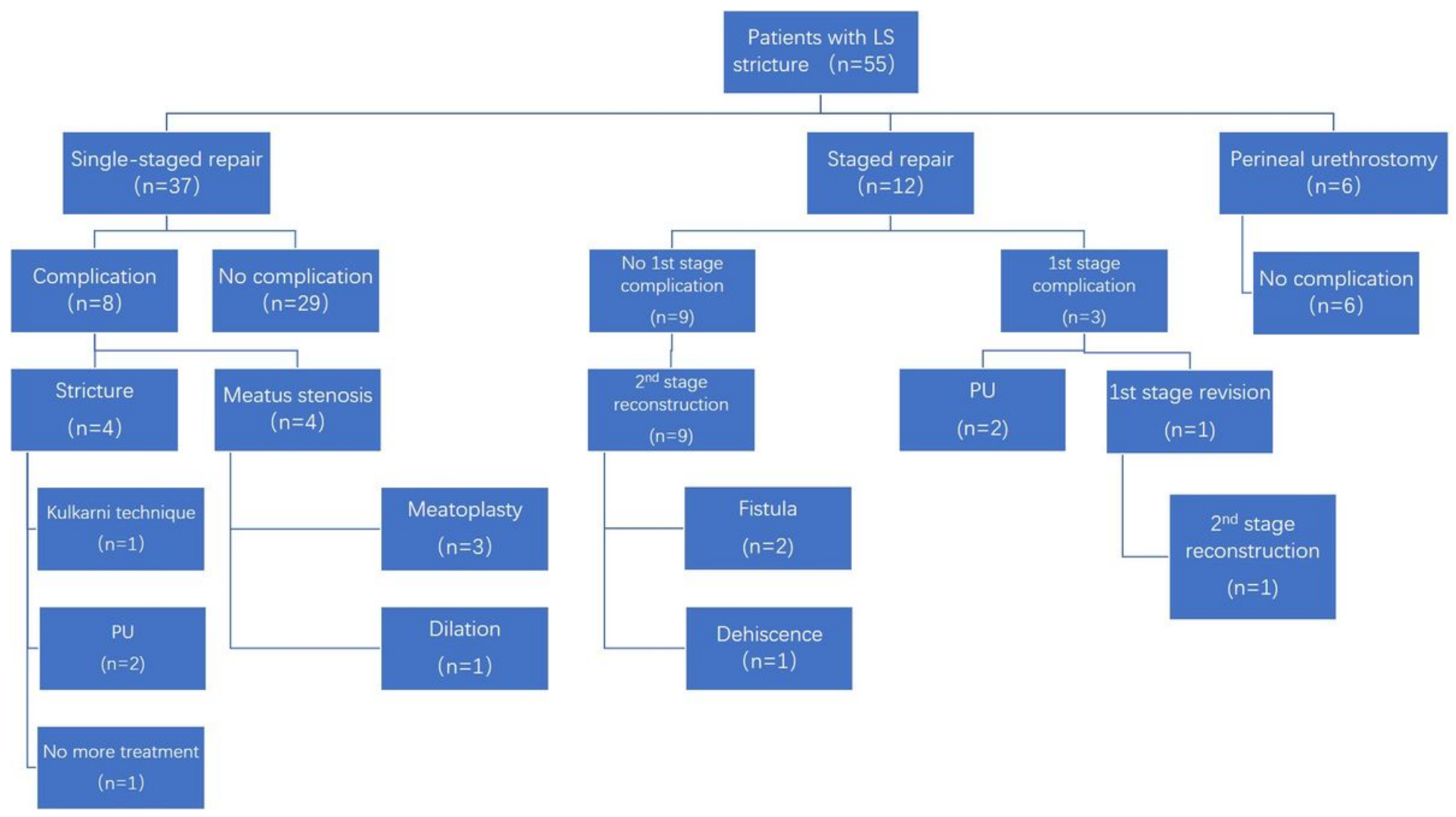

Figure 1

The evolution of three techniques in our center shows patients' original planned surgical techniques and their subsequent changes during treatment. 


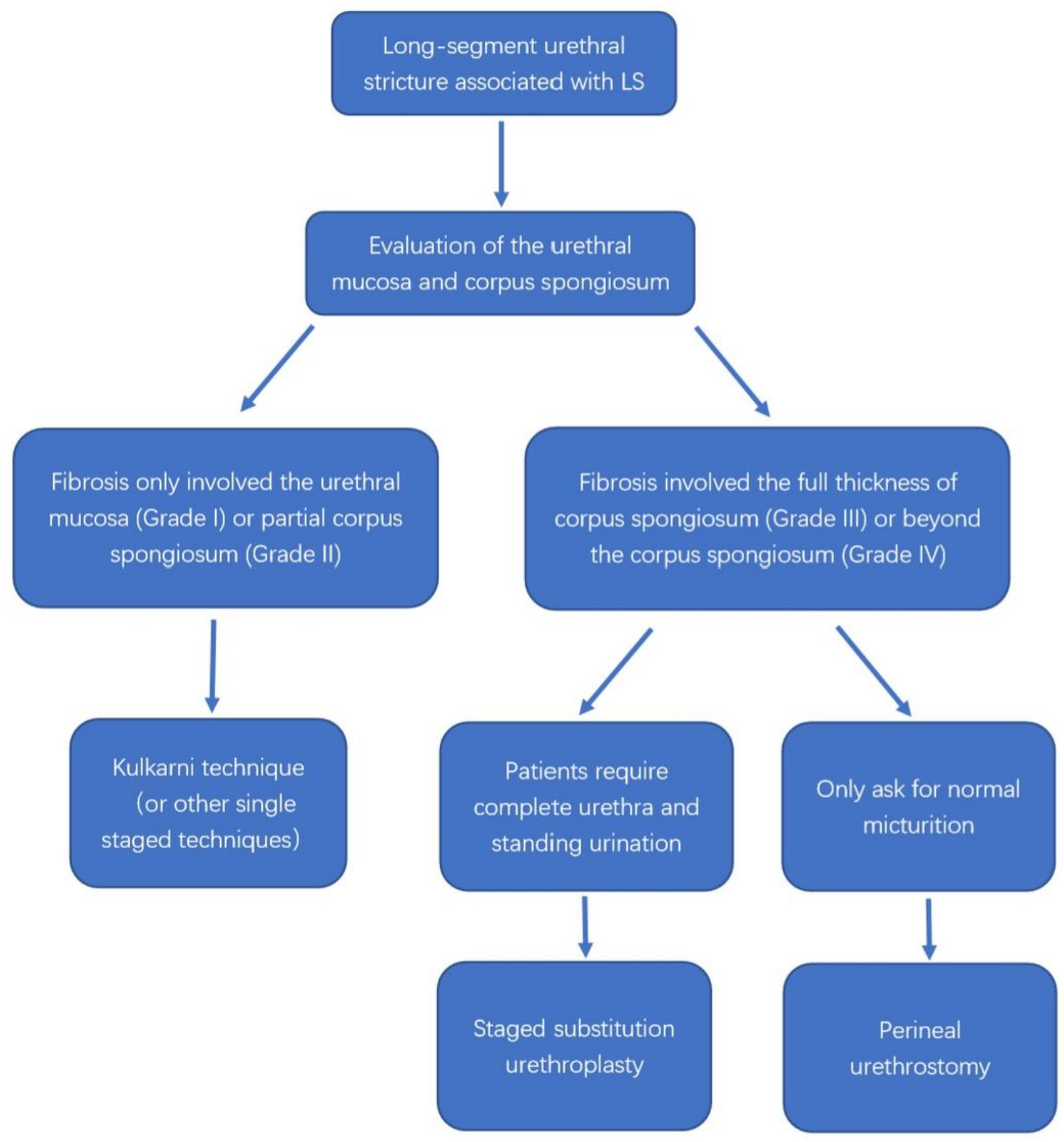

Figure 2

Our modified surgical algorithms for long-segment anterior urethral stricture associated with LS based on the evaluation of corpus spongiosum. 